\title{
Catechol-derivatized poly(vinyl alcohol) as a coating molecule for magnetic nanoclusters
}

\author{
David Burnand ${ }^{\mathrm{a}, \mathrm{b}, 1}$, Christophe A. Monnier ${ }^{\mathrm{a}, 1}$, Anthony Redjem ${ }^{\mathrm{a}, 1}$, Mark Schaefer ${ }^{\mathrm{b}, 1}$, \\ Barbara Rothen-Rutishauser ${ }^{\mathrm{a}}$, Andreas Kilbinger ${ }^{\mathrm{b}, *}$, Alke Petri-Fink ${ }^{\mathrm{a}, \mathrm{b}, * *}$ \\ a Adolphe Merkle Institute, University of Fribourg, Route de l'Ancienne Papéterie CP209, 1723 Marly 1, Switzerland \\ ${ }^{\mathrm{b}}$ Chemistry Department, University of Fribourg, Chemin du Musée 9, 1700 Fribourg. Switzerland
}

\begin{abstract}
Surface functionalization of superparamagnetic iron oxide nanoparticles (SPIONs) remains indispensable in promoting colloidal stability and biocompatibility. We propose a well-defined and characterized synthesis of a new catechol-functionalized RAFT (reversible addition-fragmentation chain transfer) poly (vinyl alcohol) polymer, which can be anchored onto hydrophobic SPIONs via a one-pot emulsion ligand exchange process. Both single and clustered nanoparticles are obtained and can be separated from each other. As clustered SPIONs are receiving increasing attention, this new macroligand might be of considerable interest for both basic and applied sciences.
\end{abstract}

\section{Introduction}

Over the past decade, nanoparticles have become increasingly prominent and particularly promising in modern-day medicine [1]. Superparamagnetic iron oxide nanoparticles (SPIONs) are forerunners in this development [2] due to their fascinating physico-chemical $[3,4]$ (i.e. superparamagnetic) properties, which make them exceptional candidates for a broad variety of clinical applications, ranging from basic contrast agents [5-8] or thermoregulators [9] to sophisticated drug delivery vectors [10-13]. Nonetheless, the therapeutic window with respect to the efficiency of these materials, in other words the size range in which these properties can be exploited, is relatively small: nanoparticles larger than approximately $20 \mathrm{~nm}$ [14] lose their superparamagnetic properties, whereas ultrasmall single nanoparticles (i.e. hydrodynamic diameter $<5.5 \mathrm{~nm}$ ) are easily cleared by the kidneys [15].

A recent strategy to circumvent this dilemma is to create large clusters of small SPIONs [16,17], thus enclosing individual superparamagnetic properties into one functional unit. It is known that

\footnotetext{
* Corresponding author.

** Corresponding author at: Adolphe Merkle Institute, University of Fribourg, Route de l'Ancienne Papéterie CP209, 1723 Marly 1, Switzerland

E-mail addresses: andreas.kilbinger@unifr.ch (A. Kilbinger), alke.fink@unifr.ch (A. Petri-Fink).

${ }^{1}$ These authors contributed equally to this work
}

iron oxide clusters present peculiar magnetic properties $[16,18]$ For example, the net magnetization is higher for SPION cluster: than for individual SPIONs $[4,18,19]$, which offers enormou: potential in therapeutics [4], magnetic resonance imaging (MRI $[16,19,20]$ and hyperthermia $[9,21]$. However, obtaining these constructs necessitates a bottom-up approach and control of the synthetic pathways and materials at a basic level, making the chemical aspects critical to creating future nanomaterials fo application-oriented purposes.

Moreover, a key point is the surface chemistry: nanomaterial: need to be firmly coated with specific polymers in order tc reinforce their colloidal stability and biocompatibility [22,23] Without them, nanoparticles tend to aggregate or be removed by the reticuloendothelial system. Several FDA-approved polymer: [24] are presently available on the market, such as polyethyleni. mine (PEI), polyvinylpyrrolidone (PVP), dextran, or polyethylent glycol (PEG), the latter being arguably the most popular polyme in pharmaceutical formulations. However, conventional PEG has : low degree of functionality. Moreover, further synthetic steps art required to increase the number of functional groups on the polymer chain end to enable subsequent chemical coupling (e.g of fluorescent dyes or targeting moieties).

In this study, we investigate a substitute custom-made polyme to provide an alternative macromolecule for surface coating. Fo this task, we synthesized poly(vinyl alcohol) (PVA), a biocompa. tible, water-soluble and non-toxic polymer [25]. To firmly anchos PVA onto the nanoparticle surface, we selectively introduced : 
catechol linker [26] at the chain end of poly(vinyl acetate) synthetized via reversible addition fragmentation transfer (RAFT), [27] as it is known that these moieties present strong affinities toward iron oxide surfaces $[8,24,28,29]$. The polymer was then coated onto monodisperse hydrophobic SPIONs coated with oleic acid via ligand exchange during an emulsion process $[18,30]$. This procedure yielded both single and clustered nanoparticles in aqueous solution. To isolate the clusters, we utilized a recently developed magnetic microreactor [31].

This approach yields a well-defined and characterized coating molecule, which can be used to obtain iron oxide nanoparticles organized in both monodisperse and clustered states. In turn, this opens the door for a broader scope of application.

\section{Material and methods}

\subsection{Materials}

AIBN (98\%), chloroform (99.8\%) hexane (>99\%), diethylether ( > 99\%), dopamine hydrochloride, ethyl-2-bromo propionate, $\mathrm{N}$ methoxycarbonylmaleimide ( $>97 \%$ ), potassium hydroxide $(85 \%)$, triethylamine (>99\%) and dichloromethane (99.9\%) were purchased from Sigma Aldrich, magnesium sulfate ( $>98 \%$ ) from SDS. Sodium hydrogen carbonate (100\%) and ethyl acetate (99.5\%) were obtained from Reactolab SA, sulfuric acid (95-97\%), methanol (99\%) and thin layer chromatography (TLC) sheets from Merck. Vinyl acetate ( $>99 \%$ ) was freshly distilled to remove polymerization inhibitors. n-hexylamine (99\%), triethylphosphite (98\%) and carbon disulfide were purchased from Acros and ethanol p.a. from VWR. Deuterated solvents ( $>99 \%$ ) were obtained from Cambridge Isotope Laboratory.

\subsection{Experimental procedures}

All reactions were carried out under inert atmosphere (argon flux). All reactants were added with a syringe. The solvent was removed by rotary evaporation at $40^{\circ} \mathrm{C}$, and compounds were dried under high vacuum on a Schlenk line. Visualization was performed with a $254 \mathrm{~nm}$ or $365 \mathrm{~nm}$ UV-vis spectrophotometer. Flash column chromatography was performed on a Biotage Isolera One chromatography system.

A first Cat-PVAc molecule of $9000 \mathrm{~g} / \mathrm{mol}$ was synthesized and used for cluster formation. As a proof of concept, another shorter Cat-PVAc of $2000 \mathrm{~g} / \mathrm{mol}$ was synthetized in order to obtain mono-isotopic resolution and was fully characterized by NMR (Fig. 1I, III and IV), gel permeation chromatography (GPC, I and III) and matrix-assisted laser desorption ionization time-of-flight (MALDI-ToF) (III).

\subsection{Polymer synthesis}

\subsubsection{RAFT agent [ethyl 2-(ethoxythiocarbonylthio)propanoate)]}

Carbon disulfide (18 g, $236.8 \mathrm{mmol}$, and 1.0 eq.) was added dropwise to a stirred solution of potassium hydroxide (13 g, $232.0 \mathrm{mmol}, 1.0$ eq.) in ethanol (100 g). After stirring for $2 \mathrm{~h}$, the solution was cooled down to $5{ }^{\circ} \mathrm{C}$ and filtered. The crude product recrystallized twice from warm ethanol in form of a yellow powder. A total yield of $24.2 \mathrm{~g}(150.9 \mathrm{mmol}, 65.0 \%)$ was quantified.

Ethyl-2-bromo propionate $(10.6 \mathrm{~g}, 58.6 \mathrm{mmol}, 1.0 \mathrm{eq}$.) in dry acetonitrile $(2 \mathrm{~mL} / \mathrm{mmol})$ was cooled down to $0{ }^{\circ} \mathrm{C}$. Potassium ethyl xanthogenate $(10 \mathrm{~g}, 62.4 \mathrm{mmol}, 1.1 \mathrm{eq}$.) was then added portionwise. The suspension was stirred at room temperature for $2 \mathrm{~h}$ before the solvent was removed under reduced pressure. After re-dissolving in dichloromethane, the organic phase was washed with Milli-Q water and saturated brine $(35.7 \% \mathrm{w} / \mathrm{w})$, dried over magnesium sulfate and concentrated in vacuo. The crude product was purified via column chromatography with a hexane:diethylether (19:1 to 9:1) mixture acting as eluent, resulting in a colorless liquid and a total yield of $8.1 \mathrm{~g}$ (36.4 mmol, 62.1\%).

\subsubsection{Catechol-maleimide linker [1-(3,4-Dihydroxyphenethyl)-1H- pyrrole-2,5-dione]}

The procedure described by Geiseler et al. [26] was modified. $\mathrm{N}$-methoxycarbonylmaleimide $(1.6 \mathrm{~g}, 10.5 \mathrm{mmol}, 1.0$ eq.) was added at $0{ }^{\circ} \mathrm{C}$ to a stirred solution of dopamine hydrochloride ( $2.0 \mathrm{~g}, 10.5 \mathrm{mmol}, 1.0$ eq.) in saturated aqueous sodium hydrogen carbonate $(60 \mathrm{~mL})$. After $20 \mathrm{~min}$, the reaction was diluted with water $(200 \mathrm{~mL})$ and stirred at room temperature for $40 \mathrm{~min}$. After acidifying to $\mathrm{pH} 1-2$ using concentrated sulfuric acid, the aqueous phase was extracted with ethyl acetate. The combined organic layers were dried and concentrated under reduced pressure ( 5 mbar). The crude product was purified by column chromatography using a dichloromethane:methanol (20:1) mixture as an eluent, leading to a yellow solid and a yield of $629 \mathrm{mg}(2.7 \mathrm{mmol}$, 25.6\%).

\subsubsection{PVAc (I)}

The procedure described by Lipscomb et al. [27] was modified. A mixture of freshly distilled vinyl acetate $(10,0 \mathrm{~g}, 116.2 \mathrm{mmol}$, $23.2 \mathrm{eq}$ ), AIBN (82.1 mg, $0.5 \mathrm{mmol}, 0.1 \mathrm{eq}$.) and ethyl 2-(ethoxythiocarbonylthio)propanoate $(1.1 \mathrm{~g}, 5.0 \mathrm{mmol}, 1.0$ eq. ) was degassed and fully immersed in a pre-heated oil bath $\left(70{ }^{\circ} \mathrm{C}\right)$. The polymerization was thermally quenched using an ice bath after $16 \mathrm{~h}$. The polymer was subsequently dissolved in dichloromethane and precipitated in a 10-fold excess of ice-cold hexane.

\subsubsection{Aminolyzed PVAc (II)}

The procedure described by $\mathrm{Ho}$ et al. [32] was modified. A mixture of poly(vinyl acetate) ( $147 \mathrm{mg}, 0.1 \mathrm{mmol}, 1.0 \mathrm{eq}$.), triethylphosphite $(0.4 \mathrm{~mL}, 2.3 \mathrm{mmol}, 28.1$ eq.) in dichloromethane $(2 \mathrm{~mL})$ was degassed. Degassed n-hexylamine ( $100 \mu \mathrm{L}, 0.8 \mathrm{mmol}, 9.8$ eq.) was added dropwise and stirred for $2.5 \mathrm{~h}$ at room temperature under inert atmosphere. The excess of $n$-hexylamine was removed by vacuum $\left(10^{-2} \mathrm{mbar}\right)$.

\subsubsection{Cat-PVAC (III)}

A mixture of 1-(3,4-dihydroxyphenethyl)-1H-pyrrole-2,5-dione (61.0 mg, $0.3 \mathrm{mmol}, 3.2 \mathrm{eq}$.$) , triethylamine (0.4 \mathrm{~mL})$ and methanol $(2 \mathrm{~mL})$ was added dropwise and the reaction stirred under Argon at room temperature overnight. The desired catechol-functionalized poly(vinyl acetate) (Fig. 1III) was purified by recycling GPC in chloroform. For this step, a Japan Analytical Industry Next System equipped with a JAIGEL $2 \mathrm{H}$ and a JAIGEL $1 \mathrm{H}$ column in series was used. The detection was performed using a UV detector at $254 \mathrm{~nm}$. $10 \mathrm{wt} \%$ solutions of the sample in chloroform were prepared and eluted with chloroform at a flow rate of $3.5 \mathrm{~mL} / \mathrm{min}$.

\subsubsection{Cat-PVA (IV)}

A mixture of Cat-PVAc ( $26.3 \mathrm{mg}, 16.0 \mu \mathrm{mol}, 1.0 \mathrm{eq}$.$) in methanol$ $(1 \mathrm{~mL})$ was added dropwise to a preheated solution $\left(65^{\circ} \mathrm{C}\right)$ of sodium hydroxide ( $120 \mathrm{mg}, 3.0 \mathrm{mmol}, 187.5 \mathrm{eq}$.$) in methanol$ $(1 \mathrm{~mL})$ and stirred overnight at constant temperature. After cooling down to room temperature, the polymer was purified by dialysis in methanol and Milli-Q water (4:1).

\subsection{SPION functionalization}

Oleic acid-coated SPIONs (average core diameter $\pm \mathrm{SD}=9.6 \pm$ $0.6 \mathrm{~nm}$, measured by transmission electron microscopy, Supp. Fig. 7A/B) synthesized by thermal decomposition [33] were mixed 
<smiles>C=COC(C)=O</smiles>

Vinyl acetate

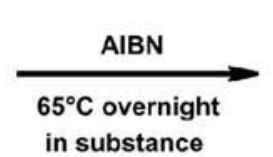

in substance

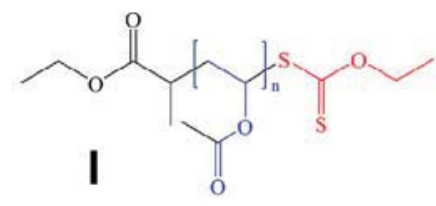

Poly(vinyl acetate)<smiles>C#C[R10](=O)OCC(C)C(=O)OCC</smiles>

Catechol-functionalized poly(vinyl acetate)<smiles>CCOC(=O)C(C)C(C)(C)CC(C)(O)SC1CC(=O)N(CCc2ccc(O)c(O)c2)C1=O</smiles>

Catechol-functionalized poly(vinyl alcohol)
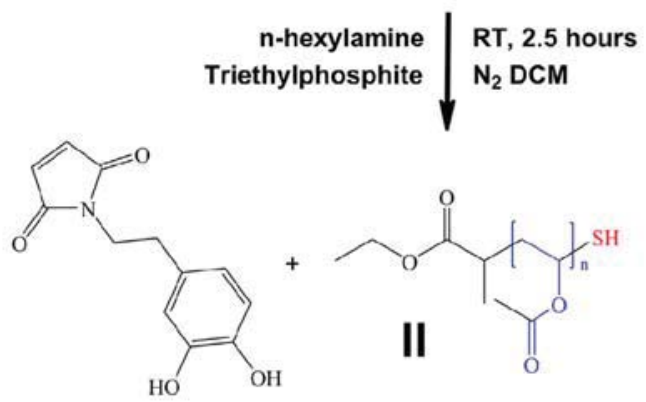<smiles>C=CCC(C)C(=O)OC(C)CC(C)(C)OC(=O)OCC</smiles>

Catechol-maleimide linker Aminolyzed poly(vinyl acetate)

Fig. 1. Catechol-functionalized PVA synthesis and subsequent SPION functionalization. I: poly(vinyl acetate) (PVAc). II: Aminolyzedpoly(vinyl acetate) (HS-PVAc') III: Catechol-functionalized poly(vinyl acetate) (Cat-PVAc). IV: Catechol-functionalized poly(vinyl alcohol) (Cat-PVA). V: Schematic representation of a Cat-PVA functionalized SPION.

with hydrophilic Cat-PVA for a ligand exchange phase transfer process in a particle:water/cat-PVA mass ratio of 10:1 (Fig. 1). The phase transfer emulsion process was carried out under magnetic stirring (750 rpm) for $12 \mathrm{~h}$ at room temperature. The aqueous phase was then collected using a pipette and diluted to $1 \mathrm{mg}$ of Cat-PVA $/ \mathrm{mL}$. The sample was then heated at $65{ }^{\circ} \mathrm{C}$ to remove turbidity. To separate undesired large aggregates from the other particles, the aqueous suspension was placed against a strong permanent magnet (Maurer Magnetic AG, Switzerland) for $12 \mathrm{~h}$. This procedure was repeated three times. The supernatant of newly functionalized nanoparticles was then transferred to a glass flask and stored at $4{ }^{\circ} \mathrm{C}$.

Magnetic purification was performed by passing the clusters through a previously developed and described microreactor [31]. They were retained within the device due to their high magnetization and subsequently collected, while single nanoparticles were collected in the flow-through fraction.

\subsection{Transmission electron microscopy (TEM) and electron tomography}

All samples were dried on regular TEM square mesh copper grids (Electron Microscopy Sciences, CF-300-Cu, carbon film) and then investigated with a Tecnai F20 transmission electron microscope (FEI). Micrographs were recorded with an UltraScan ${ }^{\mathrm{TM}} 1000$ CCD sensor (Gatan, Inc.). For electron tomography, images were subsequently acquired at sample holder tilting angles between $\pm 60^{\circ}$ with increments of $1^{\circ}$. Tomographic reconstruction (Supp.
Fig. 7D) was performed with eTomo (The Boulder Laboratory fos 3D Electron Microscopy) and rendered with 3Dmod (Version 4.5)

\section{Results and discussion}

\subsection{Polymer synthesis}

PVA-coated SPIONs have been previously used for in vitrc $[25,34,35]$ and in vivo [36] applications. However, the PVA usec in these studies do not contain any specific anchoring group. PVf is known to adsorb non-specifically onto oxide surfaces, and the interaction of the polymer with the particle surface results fron hydrogen bonding between polar functional groups of the polyme and hydroxylated and protonated surface sides of the oxide $[25,37,38]$. This relatively weak interaction may however lead tc polymer desorption from the surface once exposed to proteins ir physiological media or under high shear forces [38].

To avoid this potential problem, a maleimide catechol linke was attached to a RAFT poly(vinyl acetate). The RAFT polymeriza. tion of vinyl acetate was based on previously published report: [27]. After $17 \mathrm{~h}$ of bulk polymerization, the desired xanthate end. functionalized PVAc (Fig. 1I) was obtained. Thorough analysis by gel permeation chromatography (GPC) revealed an average mole. cular weight of $2.3 \mathrm{kDa}$, with a polydispersity of 1.2 of the synthesized polymer (See Supporting information, 7.4.). 
The thiolated form (Fig. 1II) was acquired by following a onepot xanthate moiety aminolysis under inert reducing atmosphere while using n-hexylamine and triethylphospite [32]. In ${ }^{1} \mathrm{H}-\mathrm{NMR}$, the disappearance of the methylene signal within the RAFT agent moiety at $4.7 \mathrm{ppm}$ strongly indicates a complete aminolysis (See Supporting information, 7.8.). Excess n-hexylamine was removed
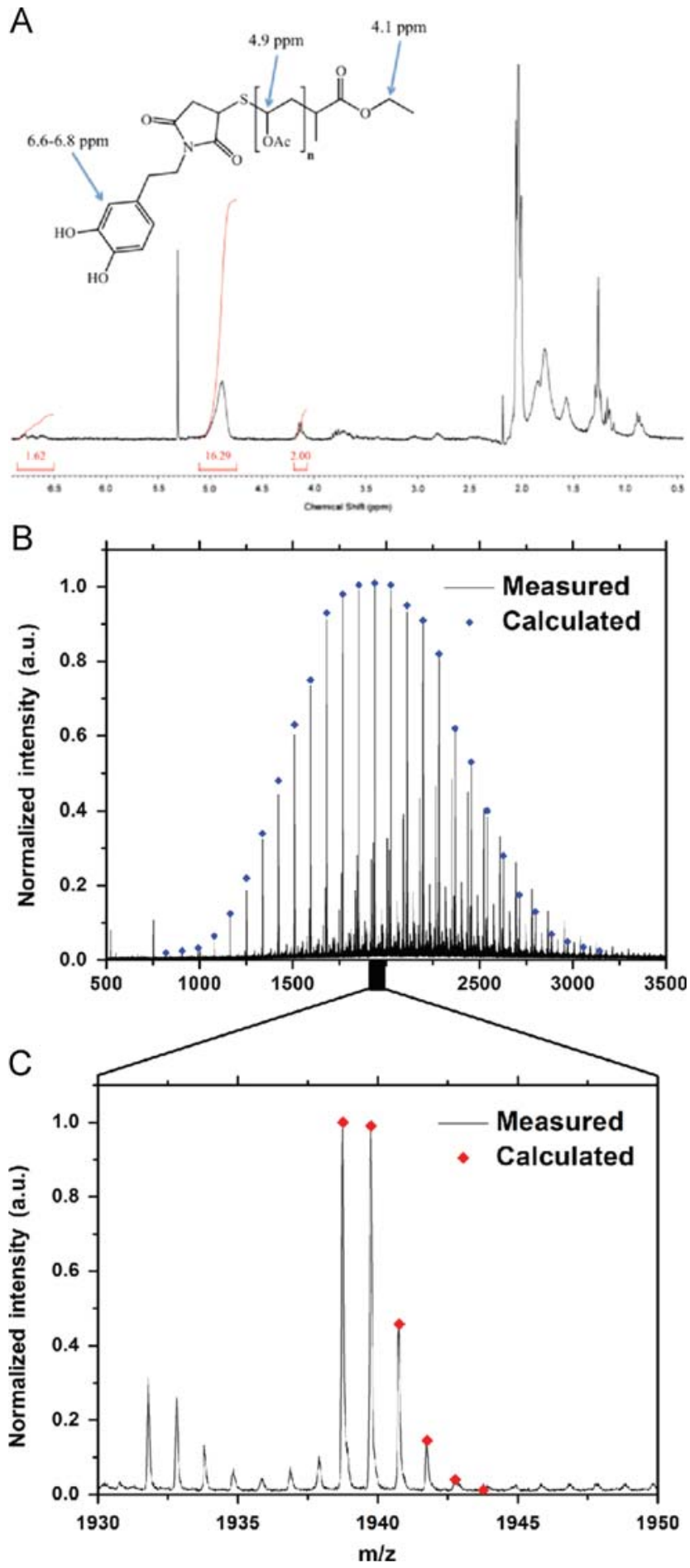

Fig. 2. ${ }^{1} \mathrm{H}-\mathrm{NMR}$ spectrum of Cat-PVAc (A) illustrating the signals used to calculate the molecular weight and degree of functionalization. MALDI-ToF mass spectrum of Cat-PVAc (B), blue squares depict the calculated position of the all $\mathrm{C}^{12}$ peaks with increasing numbers of repeat units. A zoom-in (C) on the maximum peakin monoisotopic resolution, red squares depict calculated values and isotope pattern. (For interpretation of the references to color in this figure legend, the reader is referred to the web version of this article.) in order to avoid competitive addition to the catechol-maleimide linker (structure: 1-(3,4-Dihydroxyphenethyl)-1H-pyrrole-2,5dione). The polymer was subsequently functionalized with the maleimide catechol linker [26] by 1-4 Michael addition, in which a degree of functionalization of $54 \%$ was reached (Fig 2A). The remaining non-functionalized polymer was most likely caused by disulfide coupling (SI Fig. E). Nonetheless, these disulfide species do not have any impact on the following nanoparticle coating steps, as they do not bind to iron oxide surfaces. The functional polymer was finally hydrolyzed in methanol under basic conditions and purified by dialysis.

As expected for a quasi-living polymerization technique, such as the RAFT process, the molecular weight of the polymer synthesized can be controlled by the monomer-to-CTA ratio. The molecular weight calculated by NMR $(1600 \mathrm{~g} / \mathrm{mol}$, Fig. 2A) is smaller than the target value of $2000 \mathrm{~g} / \mathrm{mol}$, thus indicating a monomer consumption of up to $70 \%$. Nevertheless, gel permeation chromatography showed a narrow molecular weight distribution, $\mathrm{PDI}=1.2$.

The molecular weight was calculated from the ratio of the methylene signal of the ethyl ester located at the beginning of each polymer chain $(4.1 \mathrm{ppm})$ and the signal of the repeat units $(4.9 \mathrm{ppm})$. Additionally, the degree of functionalization was determined by comparing the same methylene signals ( $4.1 \mathrm{ppm})$ to the aromatic signals of the catechol group (6.6-6.8 ppm). This indicated a $54 \%$ conversion of the dithioester into the catecholfunctionalized polymer (III), as shown in Fig. 2A.

MALDI-Tof/MS was used to verify the controlled synthesis of poly(vinyl acetate) (I) and its functionalization with a catechol group selectively at the chain end (III). Absolute molecular weights were determined and different species mono-isotopically resolved. The major species (Fig. 2B) can be assigned to the desired product (III) carrying an ethyl ester and a catechol end group, as well as sodium for ionization. The major peak at $1938.74 \mathrm{~g} / \mathrm{mol}$ (Fig. 2C) corresponds to a number of 18 repeat units and fits with the calculated value of $1938.76 \mathrm{~g} / \mathrm{mol}\left[\mathrm{C}_{89} \mathrm{H}_{129} \mathrm{NO}_{42} \mathrm{~S}+\mathrm{Na}^{+}\right]$. Nonetheless, there are two minor distributions that cannot be assigned to any of the intermediates or known side products such as disulfides or different oxidative states of the terminal sulfur atoms. Due to the high laser power that was needed during the measurement, it cannot be excluded that these are degradation products formed during the measurement itself.

\subsection{Cluster formation, purification and characterization}

The linking and phase transfer of hydrophobic nanoparticles primarily resulted in a heterogeneous sample of both single and clustered nanoparticles (Fig. 3A). In all, the ligand exchange and phase transfer could be conducted with an overall transfer efficiency of $50 \%$. The remaining nanoparticles were irreversible aggregates. The nanoparticle suspension remained colloidally stable in water for weeks, which is strongly indicative that the PVA was anchored to the surface. The clusters were successfully separated from the single nanoparticles (Fig. 3B/C) by the magnetic microreactor.

The clusters exhibited a systematic round shape (Fig. 3A/C, Supp. Fig. 7C) and overall diameters between 40 and $80 \mathrm{~nm}$. To investigate the architectural properties of the clusters and their nanoparticles more in detail, subsequent tilt series were acquired and used to generate a tomographic reconstruction (Fig. 3D). The three-dimensional rendering further highlights the spherical aspect and overall structure of these compounds. In all, this morphology was typical for most clusters obtained by this procedure. However, whether it is a direct consequence of the chosen surface polymer is only speculative. 

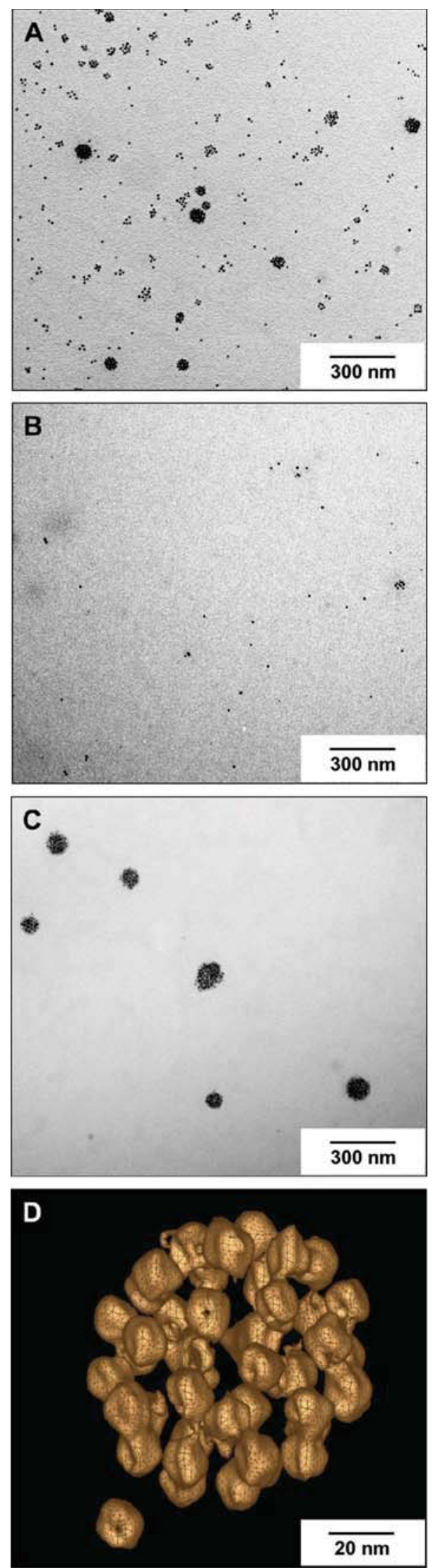

Fig. 3. Cat-PVA-SPIONs before $(A)$ and after separation $(B / C)$ visualized by TEM. Tomographic reconstruction and rendering of acquired tilt series (D) show the spatial organization of single nanoparticles within the cluster.

\section{Conclusions}

On one hand, stable and monodisperse nanoparticles are highly sought after, yet magnetic clusters are also becoming increasingly popular materials for further investigation (e.g. for MRI or mag. netic fluid hyperthermia). As a contemporary example, they are promising components of recently developed magnetic liposome: [13], which rely on well-defined and densely loaded cluster: composed of individual superparamagnetic nanoparticles.

A straightforward emulsion process was sufficient to moun this polymer onto the iron oxide nanoparticle surface. The result ing single and clustered nanoparticles remained stable in wate after ligand exchange, and could be successfully separated fron each other. The use of this new polymer led to the formation o highly uniform clusters, which has not been observed with SPION: and PVA before. With this setup, we provide a strongly linked anc well characterized catechol-functionalized PVA as an alternative tc standard and broadly used PEG, and show that this polymer is : promising candidate to obtain nanoparticle clusters. These mate rials in turn might be of interest for basic cluster studies or ever future biomedical application.

\section{Acknowledgments}

This work was supported by the Swiss National Science Foundation (126104, PP00P2-123373/1), the Adolphe Merkle Foundation and the University of Fribourg.

The authors wish to acknowledge the Dr. Alfred Bretscher Fund and access to TEM was kindly provided by the Microscopy Imagin£ Center of the University of Bern.

\section{Appendix A. Supporting information}

Supplementary data associated with this article can be found ir the online version at http://dx.doi.org/10.1016/j.jmmm.2014.09.002

\section{References}

[1] Y. Xia, Nat. Mater. 7 (2008) 758.

[2] A.K. Gupta, M. Gupta, Biomaterials 26 (2005) 3995.

[3] M. Mahmoudi, S. Sant, B. Wang, S. Laurent, T. Sen, Adv. Drug Deliv. Rev. 6 (2011) 24.

[4] S. Laurent, D. Forge, M. Port, A. Roch, C. Robic, L. Vander Elst, R.N. Mulle Chem. Rev. 108 (2008) 2064.

[5] Q.A. Pankhurst, J. Connolly, S. Jones, J. Dobson, J. Phys. D: Appl. Phys. 36 (2003 R167.

[6] J. Qin, S. Laurent, Y.S. Jo, A. Roch, M. Mikhaylova, Z.M. Bhujwalla, R.N. Mulleı M. Muhammed, Adv. Mater. 19 (2007) 1874.

[7] X. Xie, C. Zhang, J. Nanomater. 2011 (2011) 2.

[8] H. Basti, L. Ben Tahar, L. Smiri, F. Herbst, M.-J. Vaulay, F. Chau, S. Amma S. Benderbous, J. Colloid Interface Sci. 341 (2010) 248.

[9] B. Thiesen, A. Jordan, Int. J. Hyperth. 24 (2008) 467.

[10] T. Neuberger, B. Schöpf, H. Hofmann, M. Hofmann, B. Von Rechenberg, J. Magn Magn. Mater. 293 (2005) 483.

[11] U. Häfeli, Int. J. Pharm. 277 (2004) 19.

[12] M.K. Yu, Y.Y. Jeong, J. Park, S. Park, J.W. Kim, J.J. Min, K. Kim, S. Jon, Angew Chem. Int. Ed. 47 (2008) 5362.

[13] C. Bonnaud, C.A. Monnier, D. Demurtas, C. Jud, D. Vanhecke, X. Monte R. Hovius, M. Lattuada, B. Rothen-Rutishauser, A. Petri-Fink, ACS Nano \& (2014) 3451.

[14] R.M. Cornell, U. Schwertmann, The Iron Oxides structure, properties, reactions occurrence and Uses, John Wiley \& Sons, Weinheim, Germany, 2006.

[15] H.S. Choi, W. Liu, P. Misra, E. Tanaka, J.P. Zimmer, B.I. Ipe, M.G. Bawendi J.V. Frangioni, Nat. Biotechnol. 25 (2007) 1165.

[16] P. Qiu, C. Jensen, N. Charity, R. Towner, C. Mao, J. Am. Chem. Soc. 132 (2010 17724.

[17] K. Hayashi, Theranostics 3 (2013) 366.

[18] J.W. Park, K.H. Bae, C. Kim, T.G. Park, Biomacromolecules 12 (2010) 457. 
[19] C. Paquet, H.W. de Haan, D.M. Leek, H.-Y. Lin, B. Xiang, G. Tian, A. Kell, B. Simard, ACS Nano 5 (2011) 3104.

[20] L. Gao, L. Xie, X. Long, Z. Wang, C.-Y. He, Z.-Y. Chen, L. Zhang, X. Nan, H. Lei, X. Liu, Biomaterials 34 (2013) 3688.

[21] B. Mehdaoui, A. Meffre, J. Carrey, S. Lachaize, L.M. Lacroix, M. Gougeon, B. Chaudret, M. Respaud, Adv. Funct. Mater. 21 (2011) 4573.

22] M. Lattuada, T.A. Hatton, Langmuir 23 (2007) 2158.

[23] M. Mahmoudi, H. Hofmann, B. Rothen-Rutishauser, A. Petri-Fink, Chem. Rev. 112 (2011) 2323

[24] C. Boyer, M.R. Whittaker, V. Bulmus, J. Liu, T.P. Davis, NPG Asia Mater. 2 (2010)

25] A. Petri-Fink, B. Steitz, A. Finka, J. Salaklang, H. Hofmann, Eur. J. Pharm. Biopharm. 68 (2008) 129.

[26] B. Geiseler, L. Fruk, J. Mater. Chem. 22 (2012) 735.

[27] C.E. Lipscomb, M.K. Mahanthappa, Macromolecules 42 (2009) 4571.

[28] E. Amstad, A.U. Gehring, H. Fischer, V.V. Nagaiyanallur, G. Hähner, M. Textor, E.T. Reimhult, J. Phys. Chem. C 115 (2010) 683.

[29] M.D. Shultz, J.U. Reveles, S.N. Khanna, E.E. Carpenter, J. Am. Chem. Soc. 129 (2007) 2482
[30] E.D. Smolensky, H.Y.E. Park, T.S. Berquó, V.C. Pierre, Contrast Media Mol. Imag. 6 (2011) 189.

[31] R.G. Digigow, J.-F. Dechézelles, J. Kaufmann, D. Vanhecke, H. Knapp, M. Lattuada, B. Rothen-Rutishauser, A. Petri-Fink, Lab Chip 14 (2014) 2276.

[32] H.T. Ho, M.E. Levere, S. Pascual, V. Montembault, J.C. Soutif, L. Fontaine, J. Polym. Sci. Part A: Polym. Chem. 50 (2012) 1657.

[33] J. Park, K. An, Y. Hwang, J.-G. Park, H.-J. Noh, J.-Y. Kim, J.-H. Park, N.-M. Hwang, T. Hyeon, Nat. Mater. 3 (2004) 891

[34] A. Petri-Fink, M. Chastellain, L. Juillerat-Jeanneret, A. Ferrari, H. Hofmann, Biomaterials 26 (2005) 2685.

[35] V. Hirsch, C. Kinnear, M. Moniatte, B. Rothen-Rutishauser, M.J. Clift, A. Fink, Nanoscale 5 (2013) 3723.

[36] L. Crowe, F. Tobalem, A. Gramoun, B. Delattre, K. Grosdemange, J. Salaklang, A. Redjem, A. Petri-Fink, H. Hofmann, J.P. Vallée, Magn. Reson. Med. 68 (2012) 1544.

[37] L.-T. Lee, P. Somasundaran, Langmuir 5 (1989) 854.

[38] M. Chastellain, A. Petri, H. Hofmann, J. Colloid Interface Sci. 278 (2004) 353. 